\title{
High-Resolution Measurement of Resonant Wave Patterns by Perturbing the Evanescent Field Using a Nanosized Probe in a Transmission Scanning Near-Field Optical Microscopy Configuration
}

\author{
Wico C. L. Hopman, Student Member, IEEE, Remco Stoffer, and René M. de Ridder, Member, IEEE
}

\begin{abstract}
In order to model transmission scanning near-field optical microscopy (T-SNOM) experiments, we study the interaction between a nanosized atomic-force-microscopy-type probe and the optical field in a microcavity (MC) at or near resonance. Using a 2-D cross-sectional model of an experimentally studied photonic crystal MC, we have simulated the T-SNOM method by scanning a probe over the surface while monitoring the transmitted and reflected power. The simulations were performed for two probe materials: silicon and silicon nitride. From the probe-induced change in the transmission and reflection spectra, a wavelength shift was extracted. A shift almost proportional to the local field intensity was found if the resonator was excited just below a resonance wavelength. However, at the spots of highest interaction, we observed that besides the desired resonance wavelength shift, there was an increase in scattering. Furthermore, by moving the probe at such a spot in the vertical direction to a height of approximately $0.5 \mu \mathrm{m}$, a $5 \%$ increase in transmission can be established because the antiresonant condition is satisfied. Finally, a 2-D top view simulation is presented of the experimentally studied T-SNOM method, which shows a remarkably good correspondence in intensity profile, except for the exact location of the high-interaction spots.
\end{abstract}

Index Terms-Atomic-force microscopy (AFM), integrated optics, modeling, near-field microscopy, optical microcavities, optical signal detection, photonic crystal (PhC), silicon on insulator (SOI).

\section{INTRODUCTION}

$\mathbf{S}$ CANNING near-field optical microscopy (SNOM) has contributed substantially to the understanding of the effect of local geometrical features on the properties of integrated optical devices. Before, the only available method was to study a device by its reflection, transmission, or scattered power. If some unexpected behavior was observed, only simulations and a great deal of experience could be used to find an explanation for the observed phenomena. With the introduction of SNOM and all its variants, it became possible to visualize the nature of the optical modes in some photonic structures. An overview

Manuscript received December 28, 2006; revised March 23, 2007. This work was supported by NanoNed: a national nanotechnology program coordinated by the Dutch Ministry of Economic Affairs.

The authors are with the MESA+ Institute for Nanotechnology, University of Twente, 7500 AE Enschede, The Netherlands (e-mail: W.C.L.Hopman@ utwente.nl; R.Stoffer@utwente.nl; R.M.deRidder@utwente.nl).

Color versions of one or more of the figures in this paper are available online at http://ieeexplore.ieee.org.

Digital Object Identifier 10.1109/JLT.2007.897693 on the existing SNOM-related methods can, for example, be found in [1], [2], and the references therein. In present, silicon-based photonics, which are the dimensions of optical structures have shrunk down to the micrometer regime, i.e., submicrometer features are used. One of the SNOM methods to visualize light in these devices is photon scanning tunneling microscopy (PSTM), which is also sometimes referred to as near-field scanning optical microscopy (NSOM). This method has been exploited successfully on silicon-on-insulator (SOI) photonic crystal $(\mathrm{PhC})$ structures [3]-[5], which in principle are designed with periodicities around $0.5 \mu \mathrm{m}$ and hole sizes around $0.25 \mu \mathrm{m}$. For example, the method has recently been used to show slow light in W3 waveguides (i.e., three holes left out), dispersion characteristics of PhCs, and whispering gallery modes in microring resonators [4]-[6]. The disadvantage of this method and most other SNOM methods is that they cannot be used to map the resonance wave pattern in a high-quality factor $(Q)$ cavity. The reason for this is that the probe interferes too strongly with the cavity resonance; its contribution to the transfer function cannot easily be extracted from the measured signal. Both apertureless SNOM (a-SNOM; see, for example, [7]) and PSTM have this limitation because the aperture of the PSTM fiber tip has to be large enough in order to tunnel light into the probe, and for a-SNOM, the size of the probe should be large enough to be able to detect the scattered light. Another type of SNOM has recently been introduced [8] to map out the wave pattern inside a PhC microcavity (MC). This method uses the change in the transmitted optical power due to a local perturbation by a dielectric nanosized atomicforce microscopy (AFM) probe for mapping out the wave pattern inside a PhC MC. The method was later [9] termed transmission-based SNOM (or TraNSOM) but will be referred to as simply transmission-SNOM (T-SNOM) in this paper. It has been shown that $\mathrm{PhC} \mathrm{MC}$ resonances can be very sensitive to perturbations by nanoprobes [8], [10], [11]. In [8], it was suggested that the patterns found by the T-SNOM method performed on a $\mathrm{PhC} \mathrm{MC}$ were related to the local intensity of the $\mathrm{PhC}$ resonance. It was assumed that the dielectric tips mainly induced a shift in resonance wavelength. However, at the spots where the highest interaction was observed, a change in $Q$ was also observed, which could be explained from the extra out-of-plane scattering tha $t$ is locally induced by the probe. In this paper, we present a cross-sectional model, i.e., 

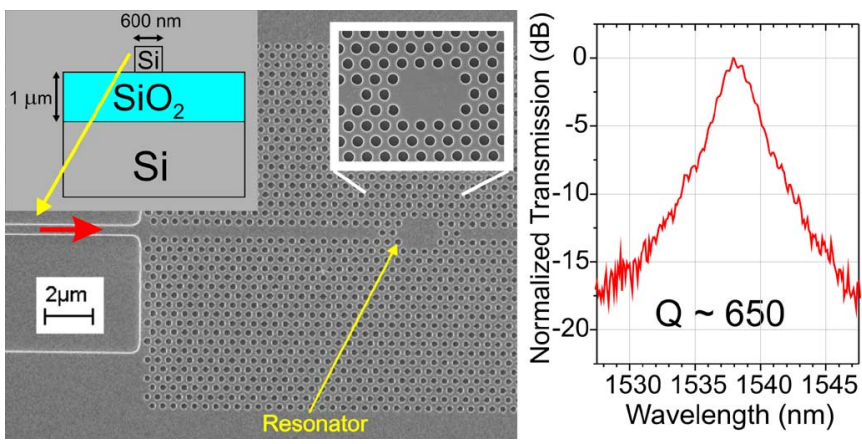

Fig. 1. (Left) SEM photo of the fabricated $\mathrm{PhC} \mathrm{MC} \mathrm{showing} \mathrm{the} \mathrm{magnification}$ of the resonator and a schematic drawing of the layer stack in the insets. (Right) Measured transmission spectrum of the $\mathrm{PhC} \mathrm{MC}$.

a waveguide grating modeled in longitudinal cross section, to verify these assumptions. After a brief description of the $\mathrm{PhC}$ $\mathrm{MC}$ and the primary measurement results in Section II, we present in Section III-A the main model and the modeling method used in this paper. The model deals with a thin $(50 \mathrm{~nm})$ silicon nitride $\left(\mathrm{Si}_{3} \mathrm{~N}_{4}\right)$ or silicon ( $\mathrm{Si}$ ) tip that is scanned over the surface of the grating, while the transmitted, reflected, and scattered powers are monitored simultaneously for each tip position. The analyses of these results are also presented in Section III-A, and the quality and practical use of the T-SNOM method will be briefly discussed. In Section III-B, we will show that besides imaging, the tip can also have the effect of suppressing the out-of-plane scattering, resulting in higher transmitted powers. The T-SNOM method as presented here is found to rely mainly on a shift in resonance wavelength. This is verified by modeling the probe as a local increase in refractive

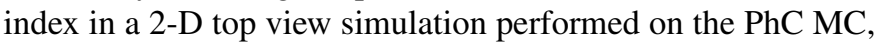
as presented in Section III-C. The main conclusions are finally summarized in Section IV.

\section{DEVICE AND MEASUREMEnTS}

The experiments reported in this section were performed on an MC integrated in a slab-type 2-D PhC in SOI (220-nm device layer thickness on $1 \mu \mathrm{m}$ of buried oxide; see Fig. 1) with hole diameters of $270 \mathrm{~nm}$ arranged in a triangular lattice with 440-nm period. A Fabry-Pérot-like cavity having a length of about $2 \mu \mathrm{m}$ was formed by placing two holes on each side of the cavity in a $\mathrm{PhC}$ waveguide.

W1 waveguides were used (i.e., one row of holes left out) as input and output waveguides for the resonator. The W1 $\mathrm{PhC}$ waveguides were connected with rib waveguides having a width of $600 \mathrm{~nm}$ for single TE-mode operation for wavelengths around $1550 \mathrm{~nm}$. The structure, as shown in the SEM photo in Fig. 1, was fabricated (at IMEC, Belgium) using a process [12] involving deep UV lithography $(\lambda=248 \mathrm{~nm})$ and reactive ion etching. A resonance wavelength of approximately $1.538 \mu \mathrm{m}$ (see Fig. 1) was measured for this PhC MC. This high-finesse cavity has a $Q$ of about 650 .

Using the T-SNOM technique with a nanosized dielectric AFM-probe, the location of the interaction pattern can be found by plotting the measured transmission versus the (2-D) tip position within the topography obtained from the cavity

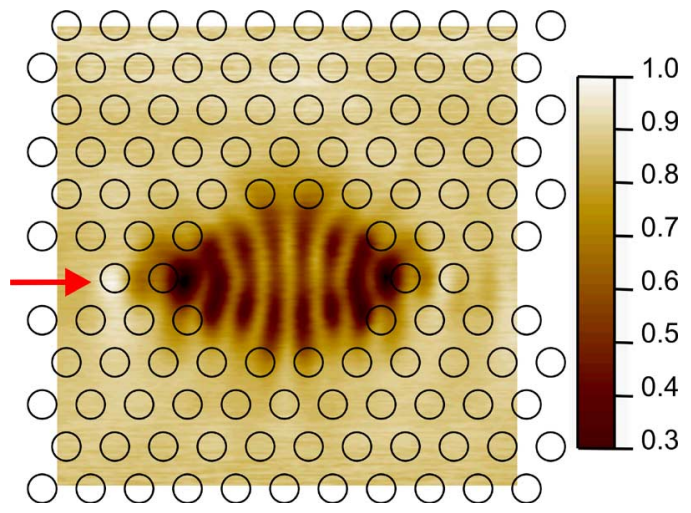

Fig. 2. Demonstration of the T-SNOM technique. The measurement was performed in contact mode (at the resonance wavelength) using a scanning probe AFM with a $\mathrm{Si}_{3} \mathrm{~N}_{4}$-probe. The red arrow indicates the propagation direction of the incident wave. The holes have been overlaid according to the position and sizes obtained from the AFM topography signal.

region. The result of the T-SNOM experiment performed at the resonance wavelength is shown in Fig. 2. A mask has been overlaid with sizes and positions according to the data obtained from the AFM height signal. To obtain this well-defined map, we used a sharpened $\mathrm{Si}_{3} \mathrm{~N}_{4}$ tip. Eight maxima can be observed with two distinct darker spots at the start and end of the cavity; the first most pronounced location (at the entrance of the cavity) is called the "hot spot" here. The measurement shows clearly that the high-interaction spots are located inside the cavity, right next to the terminating holes. A more detailed description of the measurement setup and mapping results can be found in [8].

\section{Modeling Methods}

To establish a better interpretation of the experiments described previously, 2-D simulations have been performed. In Section III-A, the side view of a comparable cross section is studied; in Section III-C, the top view is considered instead. When a silicon nitride or silicon tip is brought in close proximity to a defect cavity within a grating, i.e., perturbing the resonator with a probe, the transmission and reflection of the resonator change. The interaction may lead to a shift in resonance wavelength and an increase or even decrease in scattering, depending on the height of the probe above a field maximum in the cavity, as will be shown in Section III-B. The amount of perturbation caused by the probe is expected to be related to the local field intensity of the unperturbed resonator; the following sections will show simulations to clarify this point.

\section{A. Cross-Sectional Model}

The structure simulated in this section is a resonator formed by a defect in a 2-D waveguide grating, using the same material stack as the measured device discussed in the previous section. The grating periods and pitches were chosen to obtain a resonance near the resonance wavelength of the measured structure. The model is shown in Fig. 3.

The simulation method used is the quadridirectional eigenmode propagator (QUEP) [13]. It divides the structure into vertical slices and horizontal layers and connects slab modes of these slices and layers such that the four boundaries of the 


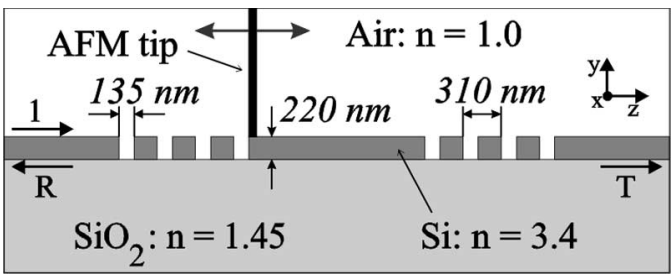

Fig. 3. Structure used in side view simulations. The black tip is either a silicon or silicon nitride tip that is scanned across the surface of the defect grating. The length of the central defect is $1.515 \mu \mathrm{m}$.

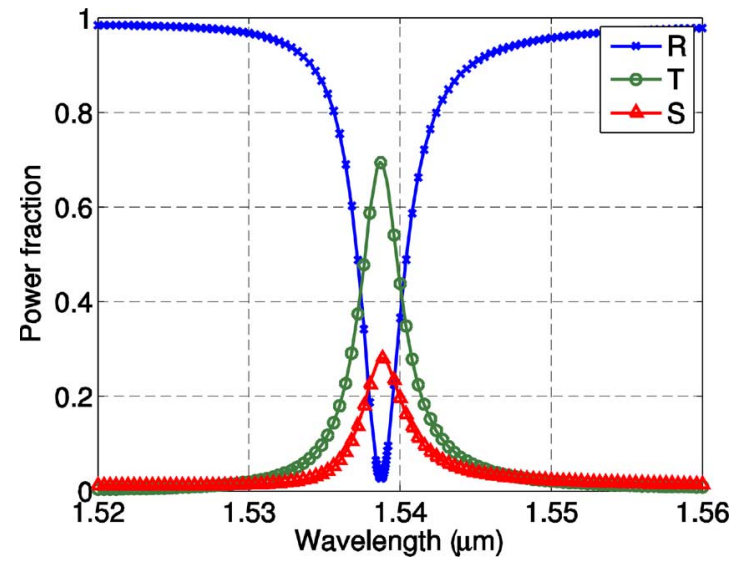

Fig. 4. Simulated spectrum of unperturbed resonator showing about $70 \%$ transmission $(T), 3 \%$ reflection $(R)$, and $27 \%$ scattering $(S=1-T-R)$ at the resonance wavelength $\left(\lambda_{\mathrm{r}}=1.5388 \mu \mathrm{m}\right)$.

computational window are transparent for outgoing radiation, while still allowing influx of light through the waveguides. It is a method that is fast for single-wavelength calculations; however, to calculate a spectrum, one needs to do calculations for each wavelength of interest.

The spectral response of the unperturbed resonator for TE polarization is shown in Fig. 4. It shows a pronounced Lorentzian-shaped resonance near $\lambda_{\mathrm{r}}=1.5388 \mu \mathrm{m}$, where the transmission rises to about $70 \%$, the reflection drops to about $3 \%$, and the remainder goes into scattering. For the experiments discussed next, we tune the wavelength either to resonance $\left(\lambda_{\mathrm{r}}\right)$ or slightly OFF-resonance at $\lambda_{\mathrm{s}}=1.5376 \mu \mathrm{m}$. Performing the T-SNOM measurements slightly OFF-resonance (preferably halfway the slope of the response curve) has the advantage of higher sensitivity to the presence of the AFM tip because the wavelength derivative of the transfer function is approximately at a maximum there. Furthermore, as will become clear later in this section, the good linear approximation to this steep slope that holds around $\lambda_{\mathrm{s}}$ turns out to be convenient for calculating the intensity profile in the resonator from the T-SNOM data.

A long rectangular-shaped silicon $(\mathrm{Si}, n=3.4)$ or silicon nitride $\left(\mathrm{Si}_{3} \mathrm{~N}_{4}, n=2.0\right)$ tip having a width of $50 \mathrm{~nm}$ is scanned across the surface of the structure. The scan path of the tip is chosen exactly parallel to the waveguide surface; it is in contact with the top of the waveguide, but it does not drop into the air holes. Fig. 5 shows the reflection and transmission for a silicon tip, at the two wavelengths $\lambda_{\mathrm{r}}$ and $\lambda_{\mathrm{s}}$. At $\lambda_{\mathrm{r}}$, we find a maximum modulation in the transmission of about $11 \mathrm{~dB}$, and for $\lambda_{\mathrm{s}}=$ $1.5376 \mu \mathrm{m}$, we find a maximum modulation of about $10 \mathrm{~dB}$. If the only effect of the perturbation by the dielectric probes

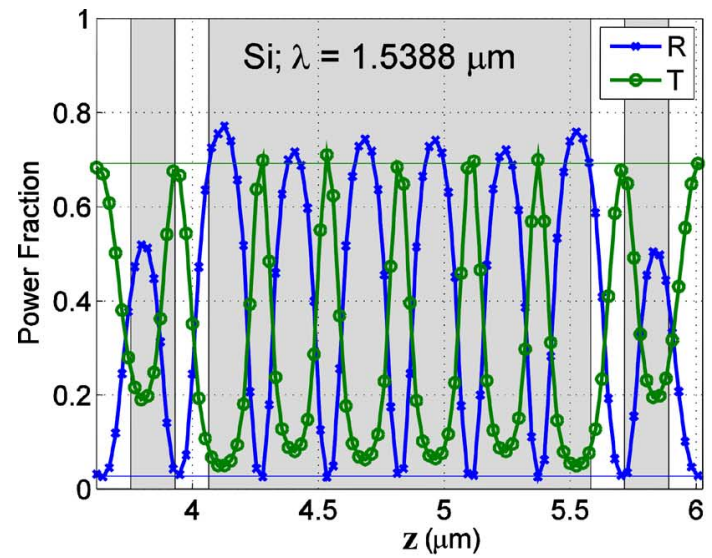

(a)

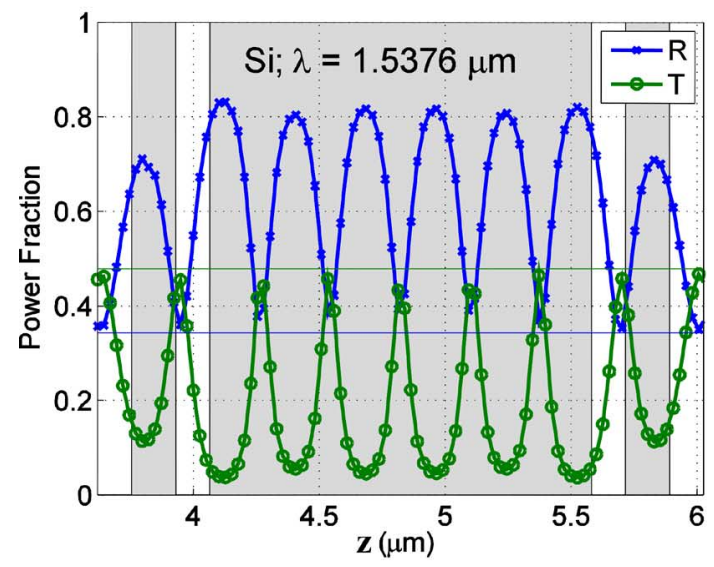

(b)

Fig. 5. Simulated power transmission and reflection for a 50-nm-wide semiinfinitely long silicon tip (probe) scanned over the cavity. The vertical white bands at both sides of the cavity indicate the positions of the holes (cf. Fig. 3). (a) At the resonance wavelength $\lambda_{\mathrm{r}}$. (b) Slightly OFF-resonance $\lambda_{\mathrm{s}}$.

would be a shift of the spectrum, the changes in reflection and transmission could be directly related to a shift of the resonance wavelength by correlating them with the spectrum in Fig. 4, as was suggested in [8]. The estimated wavelength shift derived from the transmission curve in Fig. 4 is shown in Fig. 6.

Fig. 6 shows that the silicon tip perturbs the spectrum in a slightly different way than just a wavelength shift: If the spectrum would only experience a fixed shift without additional scattering, both curves, which are calculated using reflection and using transmission, would yield the same wavelength shift because the sum of the transmission and reflection is, in that case, equal to 1 , i.e., $R+T=1$. However, due to the tip induced scattering, the transmission and reflection curves differ from the situation without probe. The induced scattering causes a difference in the observed wavelength shift (calculated from the original curves without a probe). As an example, in Fig. 7, we show the spectrum with the tip at the top of the highest disturbance $(z=4.12 \mu \mathrm{m})$ : the "hot spot." Even though the spectrum is strongly perturbed by the presence of the tip at the hot spot (compare Figs. 4 and 7), the wavelength shifts, which are calculated from transmission and reflection spectra, still show the same behavior. Their magnitude is different, but 


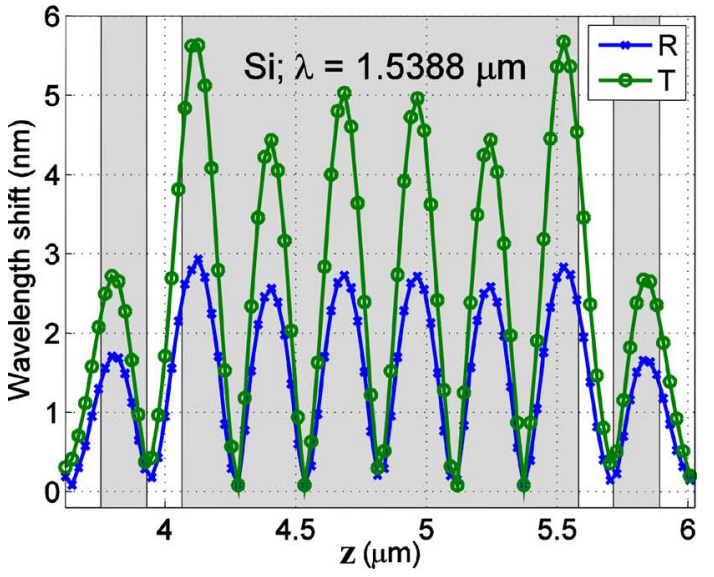

(a)

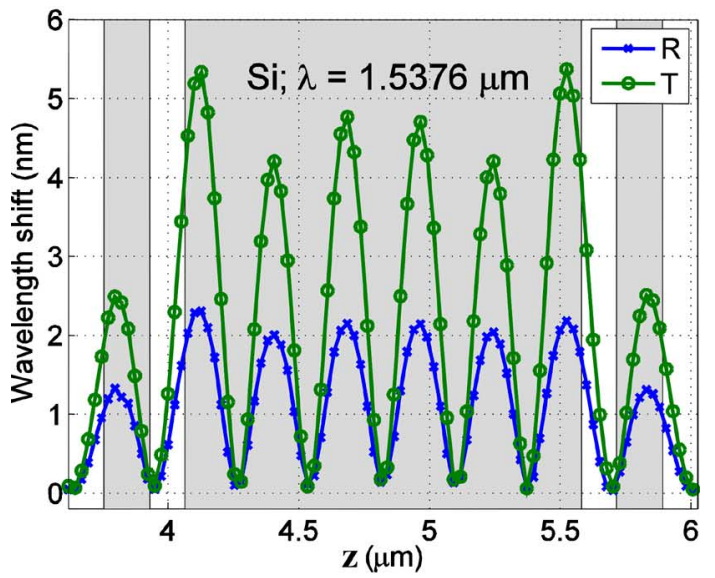

(b)

Fig. 6. Estimated wavelength shift as a function of silicon tip position, calculated by correlating either the transmission $T$ or the reflection $R$ to the unperturbed spectrum in Fig. 4. (a) Wavelength tuned ON-resonance $\left(\lambda_{\mathrm{r}}\right)$. (b) Wavelength tuned OFF-resonance $\left(\lambda_{\mathrm{S}}\right)$.

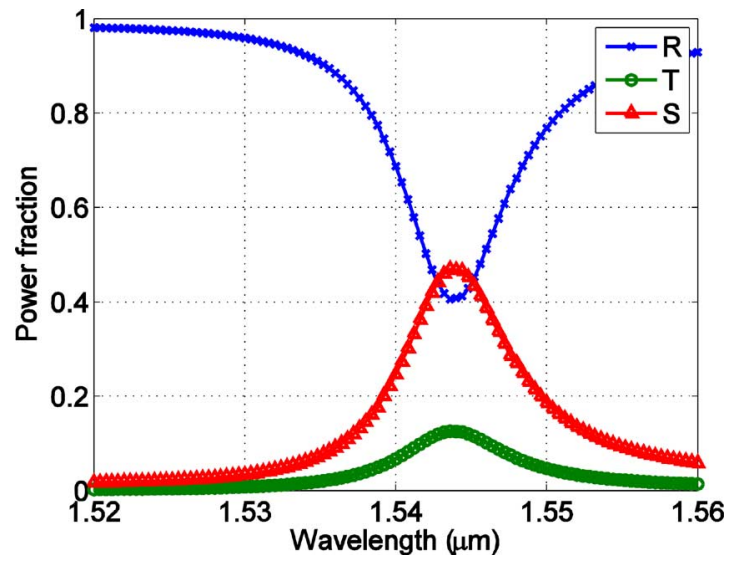

Fig. 7. Spectrum of the grating perturbed by a Si tip at the position of strongest interaction $(z=4.12 \mu \mathrm{m})$ : "the hot spot." An increased amount of scattering (cf. Fig. 4) is observed with the tip at the hot spot, which agrees with the result found in [11] and [14].

the shape is almost exactly equal, and the locations of maxima and minima match very well. The results for simulations at $\lambda_{\mathrm{r}}$ and $\lambda_{\mathrm{S}}$ only show a small difference in the magnitude of the calculated wavelength shift. From Fig. 8, it can be seen that this

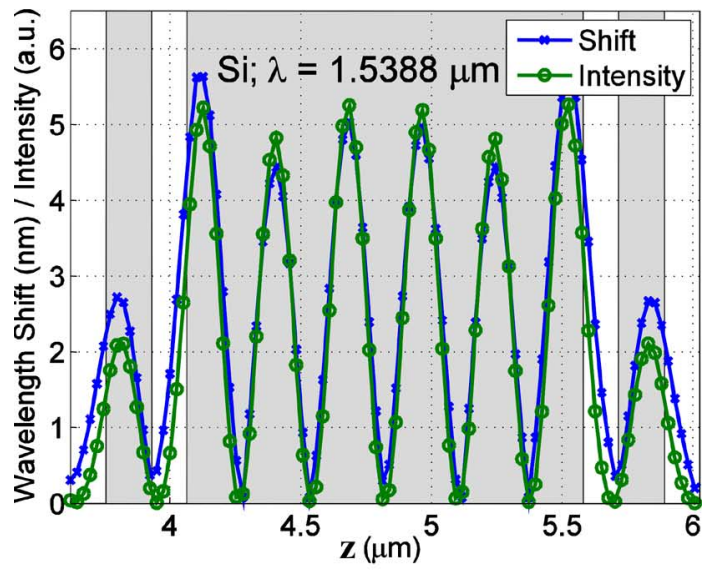

(a)

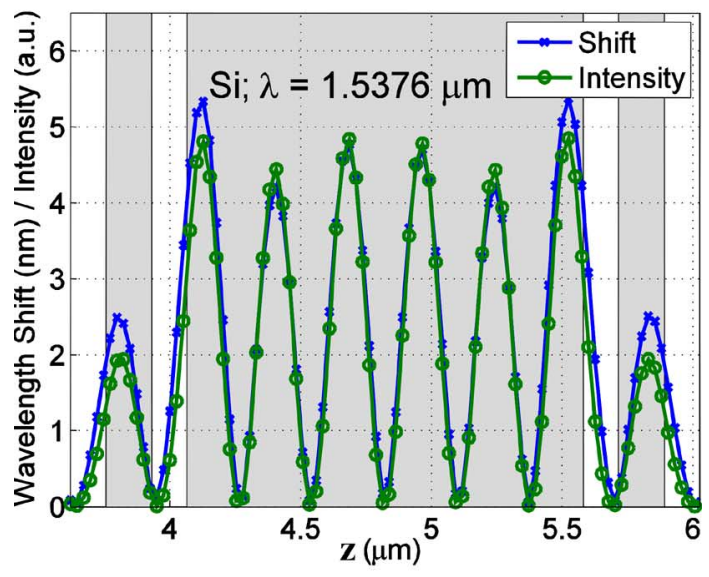

(b)

Fig. 8. Estimated wavelength shift from transmission calculation and (on the same axis) local unperturbed intensity at the silicon tip position. The intensity (at the surface) is scaled to yield good visual correspondence between the curves. Some differences are observed only at the first and last field maximum in the resonator and the maximum outside the resonator caused by probeinduced scattering. (a) $\lambda_{\mathrm{r}}=1.5388 \mu \mathrm{m}$. (b) $\lambda_{\mathrm{s}}=1.5376 \mu \mathrm{m}$.

shape correlates with the unperturbed intensity of the resonance at the surface of the resonator.

Fig. 8(a) and (b) shows the estimated wavelength shift and the intensity $\left(|E|^{2}\right)$ at the tip location, where the intensity is scaled such that the curves overlap well. These figures show that the estimated wavelength shift (calculated from the transmission curve) and the local intensity of the unperturbed resonator do indeed correlate well. Moreover, these simulations reproduce the experimentally observed feature (Fig. 2) that the hot spots are located inside the cavity and not inside the cavity terminating holes.

For the silicon nitride tip, the same procedure can be followed. The perturbance is smaller and, thus, also the wavelength shift of the resonator; moreover, the spectrum is not deformed as strongly. The unperturbed intensity (at the surface) and the apparent wavelength shift for both wavelengths are shown in Fig. 9. A similar correlation is found for $\lambda_{\mathrm{s}}=$ $1.5376 \mu \mathrm{m}$ as for the Si probe presented in Fig. 8(a) and (b). However, for the wavelength tuned to the resonance wavelength $\left(\lambda_{\mathrm{r}}=1.5388 \mu \mathrm{m}\right)$, we observe a larger difference between the wavelength shift and the local intensity. This is because 


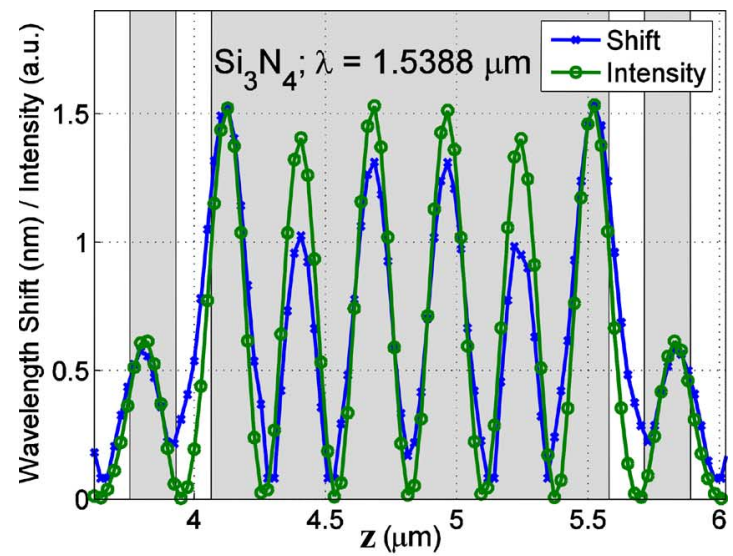

(a)

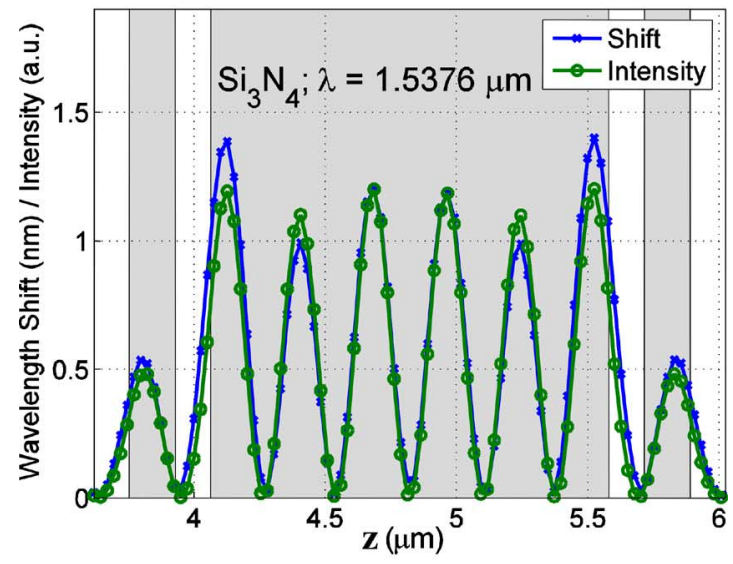

(b)

Fig. 9. Estimated wavelength shift from transmission calculation and local unperturbed intensity at the silicon nitride tip position. (a) $\lambda_{\mathrm{r}}=1.5388 \mu \mathrm{m}$. (b) $\lambda_{\mathrm{s}}=1.5376 \mu \mathrm{m}$

the spectrum is flat at the top of the curve, so the calculated wavelength shift could not be accurately determined. The optimum T-SNOM operating point is found at the wavelength where the second-order derivative is equal to zero. Obviously, when a probe with a medium or low refractive index is used, as, for example, the $\mathrm{Si}_{3} \mathrm{~N}_{4}$ probe, the choice for the operation wavelength becomes more important.

The good resemblance between the observed wavelength shifts and the intensity can be explained from the well-known [15] variation theorem, which relates a small change in dielectric constant $(\varepsilon)$ to a small change in the effective index $\left(N_{\text {eff }}\right)$. For a dielectric waveguide at a given wavelength, it can be written as

$$
\Delta N_{\mathrm{eff}}=\frac{c}{P} \iint_{\infty} \Delta \varepsilon(x, y) \mathbf{E}(x, y) \cdot \mathbf{E}^{*}(x, y) d x d y
$$

where $c$ is the speed of light in a vacuum, $\mathbf{E}$ is the modal field, and $P$ is the power carried by the mode. Equation (1) shows that the strongest effect on $N_{\text {eff }}$ can be expected at the locations of modal field maxima. In this form, the theorem holds only for waveguides with a constant cross section, so it may be safely applied only to the cavity region (not in the holes). A further condition for validity is a small probe size and/or a small $\Delta \varepsilon$

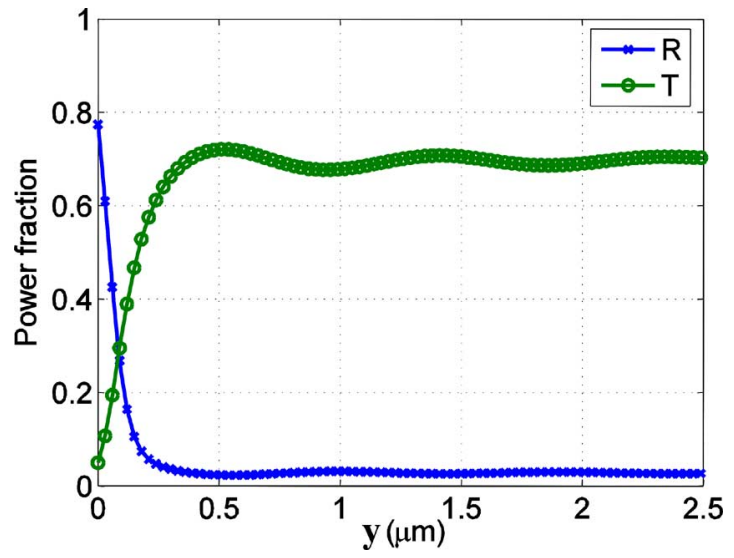

Fig. 10. As the tip is placed at the hot $\operatorname{spot}(z=4.12 \mu \mathrm{m})$ and the wavelength is set to $\lambda_{\mathrm{r}}=1.5388 \mu \mathrm{m}$, the transmission and reflection are calculated as the tip is moved vertically. At approximately a tip height $y=0.5 \mu \mathrm{m}$, the first maximum in transmission is found, which is larger than the maximum transmission without tip.

(hence small $\Delta n$ ). It is questionable whether other probes like the metallic ones used in [9] satisfy this criterion.

In conclusion, these 2-D side-view simulations indicate that the T-SNOM method can be exploited for mapping of the local intensity of the grating resonator by looking up the wavelength shifts from the transmission spectrum of the unperturbed resonator. The method only requires measuring the transmission spectrum of the unperturbed system and recording the reflected or transmitted signal while a nanosized (AFM) tip is scanned across the surface of the resonator cavity.

\section{B. Height Scan}

A decrease in the magnitude of the perturbation can be obtained by placing the silicon tip at the hot spot of the resonator in resonance, at $z=4.12 \mu \mathrm{m}$ and moving it in the upward direction $y$. The result of this simulation is given in Fig. 10 . Besides this decrease in interaction, we also observe oscillations on the transmission graph. Moreover, at approximately a tip height $y=0.5 \mu \mathrm{m}$, a maximum in transmission is found which is larger than the maximum transmission without the tip, i.e., the transmission is now about $75 \%$ instead of the $70 \%$ found without the presence of a tip. Since the oscillations are of periodic nature, as shown in the figure, the extra cavity between the resonator and the tip gives rise to this oscillation.

Two characteristic field distributions are shown in Fig. 11. Without the presence of the probe [Fig. 11(a)], we find two radiation directions above the grating (in air), having an angle $\theta$ of about $+35^{\circ}$ and $-35^{\circ}$ with respect to the normal of the waveguide plane. With the probe at height $y=0.5 \mu \mathrm{m}$, the radiation is partially suppressed [Fig. 11(b)]. If the scattered light is reflected at the probe and coupled into the guiding layer again, the following relation has to be satisfied for the antiresonance (Bragg) condition:

$$
y=\frac{\lambda(1+2 m)}{4 \cos (\theta)}, \quad m \in \mathbb{N} .
$$

Using this relation, we find the first maximum at $y \approx 0.5 \mu \mathrm{m}$ and the second one at $y \approx 1.4 \mu \mathrm{m}$, which is in good agreement 


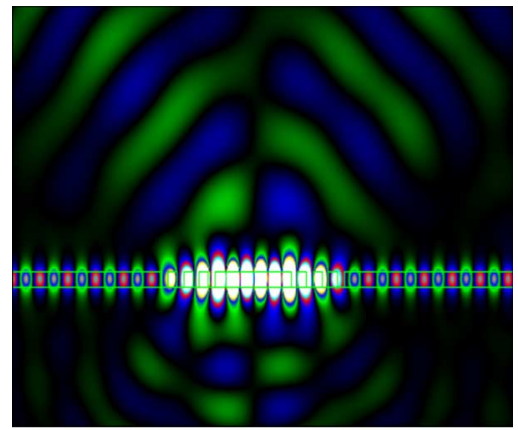

(a)

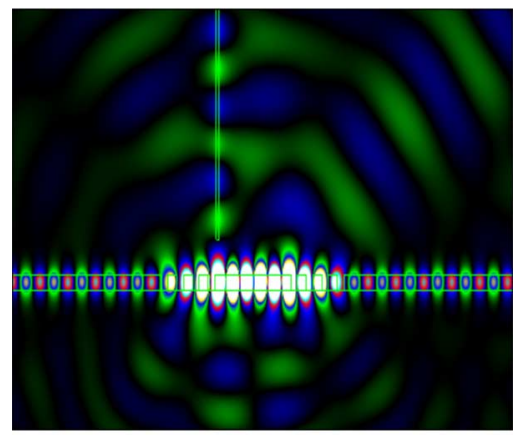

(b)

Fig. 11. (a) Modeled field distribution at the resonance wavelength $\lambda_{\mathrm{r}}$. From the figure, we derive an angle $\theta$ between the normal to the surface and the radiated beam of about $35^{\circ}$. (b) With the tip at $y=0.5 \mu \mathrm{m}$ above the surface, the disturbed field pattern shows partial suppression of the out-of-plane scattering.

with the values found in Fig. 10. These scattering suppression results are based on the same principle as is applied in hollow core waveguides [16] or 2.5-D PhCs [17], where the periodicity in the third dimension can, for example, be formed by omnidirectional Bragg mirrors. Our results show that with a small nanosized probe located at the hot spot, the transmission can be increased by $5 \%$. Even larger values can be achieved when the size of the material on top (here, the probe) is increased or when a multilayer is added to avoid the apparent leakage of light through the probe [see Fig. 11(b)].

\section{Two-Dimensional Top View Model}

In Section III-B, we showed that the intensity distribution inside a resonator could be measured indirectly by perturbation of the electric field by a dielectric probe. We found that the predominant effect of the probe is to cause a shift of the resonance wavelength and that, only at the hot spot, an increase in scattering was observed when the probe is in contact. This suggests the possibility of a kind of effective-index approximation of the PhC MC shown in Fig. 2, where the influence of "a probe" is modeled by a local increase of the refractive index. In this case, we have defined a square of 120 by $120 \mathrm{~nm}$ that simulates the probe by a local increase of the refractive index. A grid size of $40 \mathrm{~nm}$ was chosen to speed up the calculations that were performed using a finite difference timedomain (FDTD) code. The refractive index values to be used in the 2-D approximation of a $\mathrm{PhC}$ slab have been chosen in such a way that the wavelength of the calculated standing-wave pattern

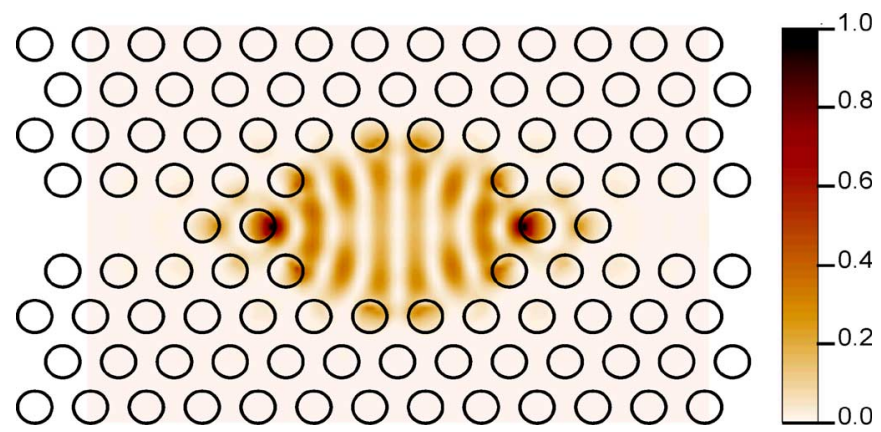

Fig. 12. Standing wave pattern in the unperturbed $\mathrm{PhC} \mathrm{MC}$, which is obtained using a 2-D FDTD simulation method displaying the square of the transverse electrical field $|\mathbf{E}|^{2}$ at the resonance.

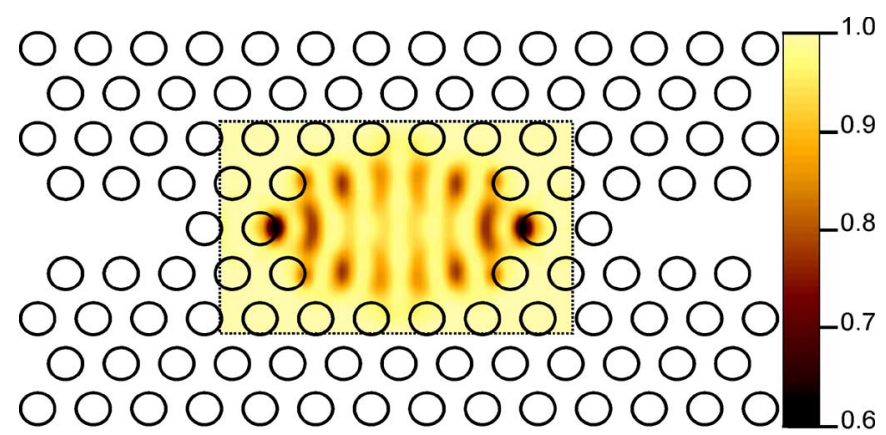

Fig. 13. Two-dimensional plot of the transmission obtained from a raster scan of a square region with a local refractive index increase of 0.2 RIU over the structure. The size of the square region was 120 by $120 \mathrm{~nm}$, and simulation grid had a size of $40 \mathrm{~nm}$.

matches the experimental observation, leading to $n_{\text {slab }}=2.9$ and $n_{\text {hole }}=1$. The top view simulations were performed such that the E-field was in the plane of the simulation (top view; see Fig. 12), and $H$-field is perpendicular to it. In a standing wave situation, the major $E$-field component has an antinode where the major $H$-field component has a node. Fig. 12 shows the intensity $|\mathbf{E}|^{2}$ in the unperturbed structure for a wavelength at resonance, excited from the left. The major difference apparent from this graph, with the measurement that was presented in Fig. 2, is in the locations of the hot spots; in the measurements, we found these locations to be right next to the two terminating holes and not inside them. This difference can possibly be attributed to the restriction enforced by the 2-D nature of the calculations. The use of a full 3-D model, like in [18], is needed to reach a better agreement in wave patterns.

As mentioned previously, we modeled the probe by an area in which the refractive index is increased by a fraction of the original index. To find the amount of refractive index units (RIU) to add to this square of 120 by $120 \mathrm{~nm}$, we first obtained the resonance with a $Q$ of about 350 by tuning of the hole radius and refractive indexes. The $Q$-value is important for the speed of the numerical experiment, since a large $Q$ would require a large simulation time for each probe position. Next, the needed increase in RIU was determined by positioning the rectangle on the hotspot found in Fig. 12 and by varying the index increase of the probe square to obtain a similar resonance wavelength shift as observed in the measurements. We found that an index increase in this square region of 0.2 RIU was sufficient. Finally, 
we conducted a 2-D parameter scan by moving the square over the region outlined by the dashed box in Fig. 13 while mapping the transmitted power. We find a remarkably good agreement between the pattern obtained from the scan and the resonance field distribution from Fig. 12. However, some minor differences are visible, particularly at the hole locations. From these modeling results, we conclude that the scanning probe can be modeled well in 2-D (top view) by a region with a slightly increased index superimposed on the original structure.

\section{CONCLUSION}

The modeling results as presented in this paper demonstrate that the T-SNOM imaging method can be used to estimate the intracavity intensity distribution of the standing wave. Using a 2-D cross-sectional model, calculated using the QUEP method, we found good agreement between the local field intensity and the simulated T-SNOM values for an operation wavelength just below the resonance wavelength, for both a silicon and a silicon nitride probe. We also observed that the probe could be used to decrease the out-of-plane scattering by moving it in the vertical direction to a height of about $0.5 \mu \mathrm{m}$ above the top surface of the structure in our case.

We also used a top-view model for calculating the intensity distribution of the standing wave in the $\mathrm{PhC} \mathrm{MC}$ using a 2-D FDTD method. We found that this calculated intensity distribution corresponds well to the pattern obtained from the measurements. However, at the hot spots, we observed a difference; the simulation suggests that we can find the highest interaction spots in the cavity-terminating holes, whereas the measurements show that these spots are located right next to these holes. Nevertheless, the simulation results obtained from the cross-sectional model agree with the experimental results that these maxima are located within the cavity and not in the cavity terminating holes.

Furthermore, we have simulated the T-SNOM method also using the top-view model of the PhC MC. The influence of the probe was calculated using a local increase in the refractive index. These T-SNOM modeling results also show good agreement with the original intensity distribution, which confirms the idea that no big error is made by neglecting the probe-induced scattering and assuming the local intensity to be proportional to the probe-induced detuning, which is the main effect determining the transmission through the structure. Therefore, we anticipate that the T-SNOM method will be a valuable tool for near-field analyses of air-clad resonators, like microring resonators, gratings, and $\mathrm{PhC}$ microcavities.

\section{REFERENCES}

[1] L. Novotny and S. J. Stranick, "Near-field optical microscopy and spectroscopy with pointed probes," Annu. Rev. Phys. Chem., vol. 57, no. 1, pp. 303-331, May 2006.

[2] S. F. Wu, "Review of near-field optical microscopy," Front. Phys. China, vol. 1, no. 3, pp. 263-274, Sep. 2006.

[3] M. L. M. Balistreri, J. P. Korterik, G. J. Veldhuis, L. Kuipers, and N. F. Van Hulst, "Quantitative photon tunneling and shear-force microscopy of planar waveguide splitters and mixers," J. Appl. Phys., vol. 89, no. 6, pp. 3307-3314, Mar. 2001.
[4] R. J. P. Engelen, T. J. Karle, H. Gersen et al., "Local probing of Bloch mode dispersion in a photonic crystal waveguide," Opt. Express, vol. 13, no. 12, pp. 4457-4464, Jun. 2005.

[5] D. J. W. Klunder, M. L. M. Balistreri, F. C. Blom et al., "Detailed analysis of the intracavity phenomena inside a cylindrical microresonator," J. Lightw. Technol., vol. 20, no. 3, pp. 519-529, Mar. 2002.

[6] H. Gersen, J. P. Korterik, N. F. van Hulst, and L. Kuipers, "Tracking ultrashort pulses through dispersive media: Experiment and theory," Phys. Rev. E, Stat. Phys. Plasmas Fluids Relat. Interdiscip. Top., vol. 68, no. 2, pp. 026604/1-026604/10, Aug. 2003.

[7] I. Stefanon, S. Blaize, A. Bruyant et al., "Heterodyne detection of guided waves using a scattering-type scanning near-field optical microscope," Opt. Express, vol. 13, no. 14, pp. 5553-5564, Jul. 2005.

[8] W. C. L. Hopman, K. O. Van Der Werf, A. J. F. Hollink et al., "Nanomechanical tuning and imaging of a photonic crystal micro-cavity resonance," Opt. Express, vol. 14, no. 19, pp. 8745-8752, Sep. 2006.

[9] J. T. Robinson, S. F. Preble, and M. Lipson, "Imaging highly confined modes in sub-micron scale silicon waveguides using transmission-based near-field scanning optical microscopy," Opt. Express, vol. 14, no. 22, pp. 10588-10595, Oct. 2006.

[10] I. Märki, M. Salt, and H. P. Herzig, "Tuning the resonance of a photonic crystal microcavity with an AFM probe," Opt. Express, vol. 14, no. 7, pp. 2969-2978, Apr. 2006.

[11] M. Hammer and R. Stoffer, "PSTM/NSOM modeling by 2-D quadridirectional eigenmode expansion," J. Lightw. Technol., vol. 23, no. 5, pp. 19561966, May 2005.

[12] W. Bogaerts et al., "Nanophotonic waveguides in silicon-on-insulator fabricated with CMOS technology," J. Lightw. Technol., vol. 23, no. 1, pp. 401-412, Jan. 2005.

[13] M. Hammer, "Quadridirectional eigenmode expansion scheme for 2-D modeling of wave propagation in integrated optics," Opt. Commun., vol. 235, no. 4-6, pp. 285-303, 2004.

[14] E. Flück, M. Hammer, A. M. Otter et al., "Amplitude and phase evolution of optical fields inside periodic photonic structures," J. Lightw. Technol., vol. 21, no. 5, pp. 1384-1393, May 2003.

[15] H. Kogelnik, "Theory of optical waveguides," in Guided-Wave Optoelectronics, 2nd ed., T. Tamir, Ed. Berlin, Germany: Springer-Verlag, 1990 , p. 31.

[16] H. P. Uranus, H. J. W. M. Hoekstra, and E. Van Groesen, "Considerations on material composition for low-loss hollow-core integrated optical waveguides," Opt. Commun., vol. 260, no. 2, pp. 577-582, 2006.

[17] B. Ben Bakir, C. Seassal, X. Letartre et al., "Room-temperature InAs/InP quantum dots laser operation based on heterogeneous ' $2.5 \mathrm{D}$ ' photonic crystal," Opt. Express, vol. 14, no. 20, pp. 9269-9276, Oct. 2006.

[18] A. F. Koenderink, M. Kafesaki, B. C. Buchler, and V. Sandoghdar, "Controlling the resonance of a photonic crystal microcavity by a nearfield probe," Phys. Rev. Lett., vol. 95, no. 15, pp. 153 904-1-153 904-4, Oct. 2005.

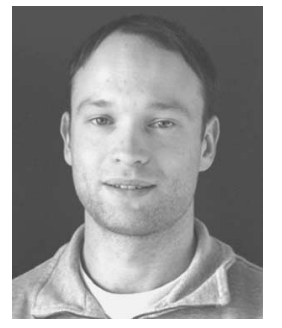

Wico C. L. Hopman (S'02) was born in Assen, The Netherlands, in 1977. He received the M.S. degree in electrical engineering from the University of Twente, Enschede, The Netherlands, in 2002 and the Ph.D. degree in the field of integrated optics in 2007.

$\mathrm{He}$ is currently working as a Researcher with the Integrated Optical Microsystems group, University of Twente. His main research interests are the fabrication and characterization methods of photonic crystal structures. 


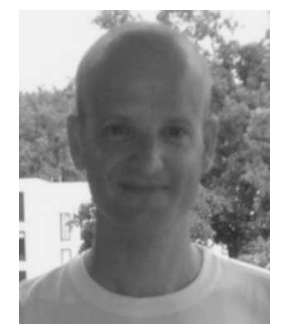

Remco Stoffer received the M.Sc. degree in applied physics and the Ph.D. degree from the University of Twente, Enschede, The Netherlands, in 1997 and 2001, respectively.

He has been with Kymata Netherlands B.V. and Alcatel Optronics Netherlands B.V. and is currently working part-time with PhoeniX B.V., The Netherlands, as a Numerical Engineer, and parttime as postdoctoral researcher at the University of Twente, working on simulations for nanomechanically actuated photonic resonators within the framework of the Dutch NanoNED project.

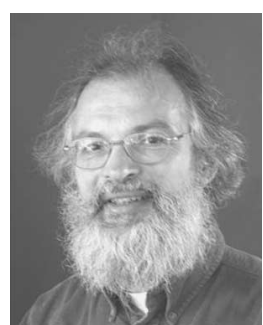

René M. de Ridder (M'94) was born 1950 in Amsterdam, the Netherlands. He received the M.S and Ph.D. degrees in electrical engineering from the University of Twente, Enschede, The Netherlands, in 1978 and 1988, respectively. His thesis subject was on sensitive thin-film magnetic-field sensors.

Since 1981, he has been a Lecturer and Research Scientist with the University of Twente, where he is currently a member of the Integrated Optical MicroSystems Group in the MESA+ Institute for Nanotechnology. In 1988, his research focus turned to integrated optical devices. A basis for his work in this field was laid during a half year of sabbatical leave at (then) AT\&T Bell Labs, Holmdel, NJ, in 1989. His current research interests include photonic crystal structures and wavelength-selective devices for applications in sensing and communication. 